\title{
Survey of Rough and Fuzzy Hybridization
}

\author{
Pawan Lingras and Richard Jensen
}

\begin{abstract}
This paper provides a broad overview of logical and black box approaches to fuzzy and rough hybridization. The logical approaches include theoretical, supervised learning, feature selection, and unsupervised learning. The black box approaches consist of neural and evolutionary computing. Since both theories originated in the expert system domain, there are a number of research proposals that combine rough and fuzzy concepts in supervised learning. However, continuing developments of rough and fuzzy extensions to clustering, neurocomputing, and genetic algorithms make hybrid approaches in these areas a potentially rewarding research opportunity as well.
\end{abstract}

\section{INTRODUCTION}

Fuzzy set theory has entered the fifth decade of research and development efforts [78]. Rough set theory celebrates its twenty-fifth anniversary this year [51]. The two theories complement each other and as such constitute important components of soft computing. Researchers have explored a variety of different ways in which these two theories interact with each other. The origins of both theories were essentially logical. Hence, much of the hybridization between fuzzy and rough set theory is logically based. Moreover, rough set theory was proposed for supervised learning. Therefore, there is a significant body of work that combines supervised classification using fuzzy and rough set theory. This paper begins with a review of fuzzy and rough hybridization in supervised learning, information retrieval and feature selection.

Both fuzzy and rough set theories are now attracting attention among researchers for a more flexible representation of clusters. There are various extensions of fuzzy and rough set theory for clustering. These include the fuzzy and rough extensions of popular clustering algorithms including Kmeans, Kohonen self-organizing maps, evolutionary unsupervised learning, and support vector clustering. In addition to a brief look at these extensions, this paper looks at attempts to combine fuzzy and rough set theory in unsupervised clustering. These research efforts include truly unsupervised hybridizations as well as semi-supervised data mining techniques that use the rule generation aspect of rough set theory.

The two theories have also made forays into black box techniques such as neurocomputing and evolutionary computing. The fuzzy and rough extensions of supervised and unsupervised neural networks have been in existence for more than a decade. Consequently, there has also been many fuzzy and rough hybridized neurocomputing work. Moreover, some innovative combinations of logical foundations of rough and

Pawan Lingras is with the Department of Mathematics and Computing Science, Saint Mary's University, Halifax, Nova Scotia, Canada, B3H3C3 (phone: +1 902420 5798; fax: +1 902420 5798; email: pawan@cs.smu.ca).

Richard Jensen is with the Department of Computer Science, The University of Wales, Aberystwyth, UK. (phone: +44 1970 621787; email: rkj@aber.ac.uk). fuzzy set theory have been integrated into neural networks. The fuzzy and rough evolutionary computing is relatively nascent. There are some examples of hybridization of fuzzy and rough sets in evolutionary computing. In some cases, the genetic algorithms are used to aid other hybridization techniques such as rough-fuzzy neural networks.

Section II provides a review of the theoretical foundations of fuzzy and rough hybridizations. The use of such hybridization in supervised learning and information retrieval is discussed in section III. Feature selection using the two theories is reviewed in section IV. Section V discusses the unsupervised learning based on combination of rough and fuzzy set theories. Fuzzy and rough extensions of black box approaches are discussed in section VI (neurocomputing) and section VII (evolutionary algorithms). Section VIII summarizes and concludes this paper.

\section{THEORETICAL HYBRIDIZATION}

There have been two main lines of thought in the hybridization of fuzzy and rough sets, the constructive approach and the axiomatic approach. A general framework for the study of fuzzy-rough sets from both of these viewpoints is presented in [77]. For the constructive approach, generalized lower and upper approximations are defined based on fuzzy relations. Initially, these were fuzzy similarity/equivalence relations [17] but have since been extended to arbitrary fuzzy relations [77]. The axiomatic approach is primarily for the study of the mathematical properties of fuzzy-rough sets [72]. Here, various classes of fuzzy-rough approximation operators are characterized by different sets of axioms that guarantee the existence of types of fuzzy relations producing the same operators.

Dubois and Prade defined the fuzzy $P$-lower and $P$-upper approximations as follows [17]:

$$
\begin{gathered}
\mu_{\underline{P} X}\left(F_{i}\right)=\inf _{x} \max \left\{1-\mu_{F_{i}}(x), \mu_{X}(x)\right\} \quad \forall i \\
\mu_{\bar{P} X}\left(F_{i}\right)=\sup _{x} \min \left\{\mu_{F_{i}}(x), \mu_{X}(x)\right\} \quad \forall i
\end{gathered}
$$

where $F_{i}$ is a fuzzy equivalence class and $X$ is the (fuzzy) concept to be approximated. The tuple $\langle\underline{P} X, \bar{P} X\rangle$ is called a fuzzy-rough set. Also defined in the literature are roughfuzzy sets [16], [63], which can be seen to be a particular case of fuzzy-rough sets. A rough-fuzzy set is a generalization of a rough set, derived from the approximation of a fuzzy set in a crisp approximation space. In [76] it is argued that, to be consistent, the approximation of a crisp set in a fuzzy approximation space should be called a fuzzy-rough set, and the approximation of a fuzzy set in a crisp approximation space should be called a rough-fuzzy set, making the two models complementary. In this framework, the approximation 
of a fuzzy set in a fuzzy approximation space is considered to be a more general model, unifying the two theories. However, most researchers consider the traditional definition of fuzzyrough sets in [17] as standard. The specific use of min and max operators in the definitions above is expanded in [57], where a broad family of fuzzy-rough sets is constructed, each member represented by a particular implicator and t-norm. The properties of three well-known classes of implicators (S-, R- and QL-implicators) are investigated. Further investigations in this area can be found in [14], [64], [74], [77].

In [9], [45], an axiomatic approach is taken, but restricted to fuzzy T-similarity relations (and hence fuzzy T-rough sets). $\mathrm{Wu}$ et al. [71] investigated the properties of generalized fuzzy-rough sets, defining a pair of dual generalized fuzzy approximation operators based on arbitrary fuzzy relations. The approach presented in [43] introduces definitions for generalized fuzzy lower and upper approximation operators determined by a residual implication. Assumptions are found that allow a given fuzzy set-theoretic operator to represent a lower or upper approximation from a fuzzy relation. Different types of fuzzy relations produce different classes of fuzzyrough set algebras.

The work in [58] generalizes the fuzzy-rough set concept through the use of residuated lattices. An arbitrary residuated lattice is used as a basic algebraic structure, and several classes of L-fuzzy-rough sets and their properties are investigated. In [11], a complete completely distributive (CCD) lattice is selected as the foundation for defining lower and upper approximations in an attempt to provide a unified framework for rough set generalizations. It is demonstrated that the existing fuzzy-rough sets are special cases of the approximations on a CCD lattice for T-similarity relations.

The relationships between fuzzy-rough set models and fuzzy $([0,1]-)$ topologies on a finite universe have been investigated. The first such research was reported in [9], where it was proved that the lower and upper approximation operators were fuzzy interior and closure operators respectively for fuzzy T-similarity relations. The work carried out in [77] investigated this for arbitrary fuzzy relations. In [56], [73] it was shown that a pair of dual fuzzy rough approximation operators can induce a topological space if and only if the fuzzy relation is reflexive and transitive. The sufficient and necessary condition that a fuzzy interior (closure) operator derived from a fuzzy topological space can associate with a fuzzy reflexive and transitive relation such that the induced fuzzy lower (upper) approximation operator is the fuzzy interior (closure) operator is also examined.

In addition to the previous approaches to hybridization, other generalizations are possible. One of the first attempts at hybridizing the two theories is reported in [75], where rough sets are expressed by a fuzzy membership function to represent the negative, boundary and positive regions. All objects in the positive region have a membership of one and those belonging to the boundary region have a membership of 0.5 . Those that are contained in the negative region (and therefore do not belong to the rough set) have zero membership. In so doing, a rough set can be expressed as a fuzzy set, with suitable modifications to the rough union and intersection operators. In [46], a definition of fuzzyrough sets is given based on an algebraic approach to rough sets proposed by Iwinski, where a rough set is defined as a pair of subsets from a sub-Boolean algebra without reference to the universe. The lower and upper bounds of an Iwinski rough set are then fuzzified. As stated in [76], the precise meaning of the upper and lower bounds may not be clear.

Another approach that blurs the distinction between rough and fuzzy sets has been proposed in [53]. The research was fuelled by the concern that a purely numeric fuzzy set representation may be too precise; a concept is described exactly once its membership function has been defined. It seems as though excessive precision is required in order to describe imprecise concepts. The solution proposed is termed a shadowed set, which does not use exact membership values but instead employs basic truth values and a zone of uncertainty (the unit interval). This can be thought of as an approximation of a fuzzy set or family of fuzzy sets where elements may belong with certainty (membership of 1), possibility (unit interval) or not at all (membership of 0 ). This can be seen to be analogous to the rough set definitions for the positive, boundary and negative regions.

\section{SUPERVISED LEARNING AND INFORMATION RETRIEVAL}

In [59] a fuzzy-rough nearest neighbor classification approach is presented that attempts to handle both fuzzy uncertainty (caused by overlapping classes) and rough uncertainty (through insufficient features). A fuzzy-rough ownership function is constructed that attempts to incorporate these two factors, and also allows a possibilistic class membership assignment. All training patterns influence the ownership function, and hence no decision is required as to the number of neighbors to consider, although there are other parameters that must be defined for its successful operation. It should be noted that the algorithm does not use fuzzy lower or upper approximations to determine class membership.

This work is extended in [67]. Here, the classification task is divided into four stages. First, the fuzzy-rough ownership is calculated (via a leave-one-out strategy) for each training pattern in the dataset for all classes. This value indicates the degree to which other patterns support each data item. Next, the training set is filtered to eliminate inconsistencies - where the highest fuzzy-rough ownership value for a data item suggests a class other than the known class. Then, representative points are selected from the processed training set and fuzzy-rough ownership values updated based on mountain clustering. Finally, test patterns are classified using only the representative points determined previously, using the fuzzy-rough algorithm in [59]. Bian and Mazlack [8] incorporate rough uncertainty into the fuzzy K-NN classifier through the use of fuzzy upper and lower approximations as defined in [17]. The $\mathrm{K}$ nearest neighbors are used to determine the membership degree of the test pattern to the fuzzy lower and upper approximations for every class. 
The induction of gradual decision rules, based on fuzzyrough hybridization, is given in [19], [20]. For this approach, new definitions of fuzzy lower and upper approximations are constructed that avoid the use of fuzzy logical connectives altogether, and hence bypass this arbitrary choice. Decision rules are induced from lower and upper approximations defined for positive and negative relationships between credibility of premises and conclusions. In addition to this, a new scheme of inference is proposed based on a generalized fuzzy-rough modus ponens.

Fuzzy decision tree induction is an area of much interest, due mainly to its ability to model vagueness, and seems a logical area of application for fuzzy-rough sets. However very little work has been carried out as yet. Initial research is presented in [5] where a method for fuzzy decision tree construction is given that employs the fuzzy-rough ownership function from [59]. This is used to define both an index of fuzzy-roughness and a measure of fuzzy-rough entropy as a node splitting criterion. Traditionally, fuzzy entropy (or its extension) has been used for this purpose. In [31], a fuzzy decision tree algorithm is proposed, based on fuzzy ID3, that incorporates the fuzzy-rough dependency function defined in [29] as a splitting criterion. It is shown that the fuzzyrough method performs comparably to fuzzy ID3 for fuzzy datasets, and better than it for crisp data. A fuzzy-rough rule induction method is proposed in [23] for generating certain and possible rulesets from hierarchical data.

Fuzzy-rough ownership is used to measure the average ruggedness of data for time series in [60]. The rate of average fuzzy-roughness change is examined when the resolution of the time series is changed. By this method, a fractal dimension that quantifies the regularity of the time series is determined. The proposed dimension is relatively insensitive to translation, scaling, noise and non-stationarity.

A characteristic of natural language is its inherent vagueness and uncertainty, and hence is an ideal area of application for fuzzy-rough set hybrids. In [15], an approach to web query expansion is proposed using fuzzy-rough set theory as a framework for query refinement. A thesaurus for this task, defining an approximation space, can be approximated from the upper and the lower side. The use of fuzzy-rough sets is motivated by the extra flexibility afforded by graded thesauri and weighted queries - a thesaurus may be represented as a fuzzy relation and the query as a fuzzy set. Query expansion and refinement occurs through the use of fuzzy lower approximations of fuzzy upper approximations of the query itself. Automatic text summarization is a process which condenses a source document into a much shorter text with the aim of retaining its core content. The authors in [28] propose a fuzzy-rough set aided summarization method to extract key sentences from a text as a document summary. Sentences are given a relevance ranking based on fuzzyrough approximations based on a fuzzy relevance clustering.

\section{Feature Selection}

One of the primary and most successful applications of crisp rough sets is to the area of feature selection. It is natural, then, to apply its hybridization to this area [33]. Such work has been carried out in [29], [32], [61], where a reduction method was proposed based on fuzzy extensions to the positive region and dependency function based on fuzzy lower approximations. A greedy hill-climber is used to perform subset search, using the fuzzy dependency function both for subset evaluation and as a stopping criterion. The method was used successfully within a range of problem domains, including web content classification and complex system monitoring [32].

Optimizations are given in [6], [32] to improve the performance of the method. In [7], a compact computational domain is proposed to reduce the computational effort required to calculate fuzzy lower approximations for large datasets, based on some of the properties of fuzzy connectives. Fuzzy entropy is used in [41] to guide the search toward smaller reducts. In [6], an alternative search algorithm is presented that alleviates some of the problems encountered with a greedy hill-climber approach. This problem is also tackled in [30] via the use of a novel ant colony optimization-based framework for feature selection. A genetic algorithm is used in [81] for search based on the fuzzy dependency function within a face recognition system with promising results.

The work in [65], [68] improves upon these developments by formally defining relative reductions for fuzzy decision systems. A discernibility matrix is constructed for the computation of all such reductions. As the resulting discernibility matrix is crisp, some information may have been lost in this process. Additionally, there are complexity issues when computing discernibility matrices for large datasets. However, in the crisp rough set literature there have been methods proposed that avoid this, and similar constructions may be applicable here.

Feature selection algorithms, based on the generalization of fuzzy approximation spaces to fuzzy probability approximation spaces, are introduced in [26]. This is achieved through the introduction of a probability distribution on the universe. Information measures for fuzzy indiscernibility relations are presented in [25] for the computation of feature importance. Reducts are computed through the use of a greedy selection algorithm.

\section{UNSUPERVISED LEARNING AND CLUSTERING}

Rough set theory has made substantial progress as a classification tool in data mining [52]. The basic concept of representing a set as lower and upper bounds can be used in a broader context. Clustering in relation to rough set theory is attracting increasing interest among researchers [22], [54], [66]. One of the first proposals of unsupervised learning in the context of rough set theory can be found in 1996 [35], where the concept of lower and upper bounds of numeric ranges was used to create a rough Kohonen neural network. A similar concept was also used for the clustering of traffic patterns based on rough genetic algorithms [37]. These early proposals did not exploit the set theoretic concept of lower and upper bounds. The rough set representation of clusters can be found in [36], which described how a rough 
set theoretic classification scheme can be represented using a rough set genome. In subsequent publications [39], [38], modifications of K-means and Kohonen Self-Organizing Maps (SOM) were proposed to create intervals of clusters based on rough set theory. Recently, [70] showed how K nearest neighbour classification can be augmented with rule induction. Asharaf, et al. [3] created rough boundaries around irregular shaped clusters based on support vector clustering.

Unsupervised clustering based on fuzzy set theory can be traced back more than twenty-five years to Bezdek's proposal of fuzzy c-means algorithm [4]. Hoppner et al. [24] provide a comprehensive overview of fuzzy clustering. The fuzzy clustering techniques discussed in their book include the fuzzy c-means, the Gustafson-Kessel and the Gath-andGeva algorithm for classification problems. There have been a few studies that compare, complement, and combine rough and fuzzy set based clustering. Lingras [40] compared the results of fuzzy clustering that included creation of rough set representation of clusters using fuzzy memberships from the fuzzy c-means algorithm. Asharaf and Murty [2] describe a hybridized fuzzy-rough approach to clustering. Chimphlee, et al. [12] described how feature selection based on independent component analysis can be used for hybridized rough-fuzzy clustering of web user sessions.

There are a number of approaches that combine the traditional supervised classification from rough set theory with unsupervised fuzzy clustering. For example, [80] use a rough set-based fuzzy clustering algorithm in which the objects of fuzzy clustering are initial clusters obtained in terms of equivalence relations. The preponderance of many small classes is countered through secondary clustering on the basis of defining fuzzy similarity between two initial clusters. Wang, et al. [69] integrated fuzzy clustering and variable precision rough set theory. This approach was used to effectively discover association rules in process planning.

Traditional hybridization of fuzzy and rough sets can also be seen in [55], where a texture separation algorithm is proposed to solve the problem of unsupervised boundary localization in textured images using rough fuzzy sets and hierarchical clustering. Pal [47] in a book chapter describes how rough-fuzzy initialization can be used for clustering with the example of multi-spectral image segmentation. In another chapter in the same book, he describes how rough set-based rule extraction can be combined with self-organizing maps.

\section{NEUROCOMPUTING}

While most of the rough-fuzzy hybridization efforts are focused on classification, such hybridization is finding increasing popularity in neurocomputing. Use of rough set theory in neurocomputing is relatively recent, when in 1996, Lingras [34], [35] showed how upper and lower bounds of ranges of numbers may be able to reduce training time and improve prediction performance. Lingras [36] also showed how rough neurons can be combined with neo-fuzzy neurons.

A slightly different knowledge-based approach can be found in a book by Pal and Mitra [48] where they report the usefulness of rough and fuzzy hybridization in knowledgebased networks. Han et al. [21] used a combination of roughfuzzy neural computing for classifying faults in high voltage power systems. They also showed how the combination of rough and fuzzy sets compares favorably with fuzzy neural computing. Pal et al. [49] used an innovative combination of multiple technologies including rough sets, fuzzy sets, neurocomputing, and genetic algorithms that provided accelerated training and a compact network suitable for generating a minimum number of rules with high certainty values. Zhang [79] combined the logical view of rough set theory with fuzzy neurocomputing. Rough set theory was used to reduce the rule set in the initial fuzzy system by eliminating inconsistent and redundant rules. The resulting rule set is used to create and train a simple fuzzy neural network. A similar hybridization can also be found in [27], where particle swarm optimization is used to refine network parameters.

The traditional rough set strength of rule set management was combined with neuro-fuzzy systems by [1] for time series prediction based on real world stock market data. The proposed model creates rules with reasonable interpretability and leads to higher yields. The use of upper and lower bounds of numeric ranges is also shown to be effective in classification of multi-spectral images in [42], where a rough-fuzzy Hopfield net (RFHN) is proposed. The approach used upper and lower bounds of gray levels captured from a training vector in multi-spectral images instead of all the information in the image. The resulting RFHN reduces training time due to use of $2 / \mathrm{N}$ pixels for an $\mathrm{N}$-dimensional multispectral image. The resulting classification is also shown to be an improvement over the conventional approach. A similar improvement in performance with the use of rough-fuzzy neural networks over fuzzy neural networks was reported in [18] for prediction of short-term electricity load forecasting. They also used genetic algorithms for the selection of the best set of inputs for the prediction. The application of roughfuzzy neurocomputing continues to diversify as indicated by the rough-fuzzy neural network controller used by [10] for waste water management.

\section{Evolutionary AND Genetic Algorithms}

There is a fifteen year history of hybridization of fuzzy and genetic algorithms (GAs). The first ten year history of such hybridization is reported in [13]. Hybridization of GAs with rough set theory was first based on lower and upper bounds of numeric ranges in the form of rough genetic algorithms in 1998 [37]. Another hybridization between GAs and rough set theory used rough sets representation in the genetic encoding [39] for creating rough set representations of clusters. Mitra and Mitra [44] proposed a hybrid decision support system for detecting the different stages of cervical cancer. Hybridization included the evolution of knowledgebased subnetwork modules with GAs using rough set theory and the ID3 algorithm.

Genetic algorithms have been used with hybridized rough and fuzzy neural research by a number of authors. For example, [18] used GAs for selecting the best set of inputs 
for rough-fuzzy neurocomputing. Similarly, [49] used GAs in rough and fuzzy set based rule generation. These genetic algorithms were not specifically extended based on either rough or fuzzy set theory. One example of an extended GA based on rough set theory combined with fuzzy set theory can be found in [62]. The authors applied rough GAs based on lower and upper bounds of numeric ranges to the classification problem in an undetermined environment based on a fuzzy distance function by calculating attribute weights.

\section{SUMMARY AND CONCLUSIONS}

This paper reviewed fuzzy and rough hybridization efforts. The review can be broadly categorized into logical and black box approaches. The logical approaches can be further subdivided into theoretical, supervised learning, feature selection, and unsupervised learning. The black box approaches consist of neural and evolutionary computing. There is significant theoretical work on hybridization of fuzzy and rough set theories, as well as its usage in classification and similar supervised learning techniques. There is also an increasing interest in extension of these two theories in unsupervised clustering and in semi-supervised learning.

Neurocomputing extensions of fuzzy and rough set theory have been in existence for more than a decade. The hybridization of fuzzy and rough set theories can be seen in the neurocomputing world at various levels. These two theories not only provide different modeling of input and output neurons, but are also used for introducing logical reasoning in neural networks. While rough and fuzzy extensions of genetic algorithms are being proposed, the use of hybridized rough and fuzzy sets in genetic computing is relatively limited. However, a number of researchers have used evolutionary computing in the more traditional logical hybridization of these two theories. Since fuzzy and rough set theory complement each other, one expects further work in their hybridization in all of the areas discussed in this paper.

\section{ACKNOWLEDGMENTS}

This work is partly funded by the UK EPSRC grant GR/S98603/01.

\section{REFERENCES}

[1] K.K. Ang and C. Quek, "Stock trading using RSPOP: a novel rough setbased neuro-fuzzy approach," IEEE Transactions on Neural Networks, vol. 17 , no. 5, pp. 1301-1315, 2006.

[2] S. Asharaf and M.N. Murty, "An adaptive rough fuzzy single pass algorithm for clustering large data sets," Pattern Recognition, vol. 36, no. 12 , pp. 3015-3018, 2004.

[3] S. Asharaf, S.K. Shevade, and N.M. Murty, "Rough Support Vector Clustering," Pattern Recognition, vol. 38, no. 10, pp. 1779-1783, 2005.

[4] J. C. Bezdek, Pattern Recognition with Fuzzy Objective Function Algorithms, Plenum:NY, 1981.

[5] R.B. Bhatt and M.Gopal, "FRID: Fuzzy-Rough Interactive Dichotomizers," IEEE International Conference on Fuzzy Systems (FUZZIEEE'04), pp. 1337-1342, 2004

[6] R.B. Bhatt and M. Gopal, "On fuzzy-rough sets approach to feature selection," Pattern Recognition Letters, vol. 26, no. 7, pp. 965-975, 2005

[7] R.B. Bhatt and M. Gopal, "On the compact computational domain of fuzzy-rough sets," Pattern Recognition Letters, vol. 26, no. 11, pp. 1632-1640, 2005.
[8] H. Bian and L. Mazlack, "Fuzzy-Rough Nearest-Neighbor Classification Approach," Proceeding of the 22nd International Conference of the North American Fuzzy Information Processing Society (NAFIPS), pp. 500-505, 2003.

[9] D. Boixader, J. Jacas, and J. Recasens, "Upper and lower approximations of fuzzy sets," International Journal of General Systems, vol. 29, no. 4, pp. 555-568, 2000.

[10] W.C. Chen, N.B. Chang, and J.C. Chen, "Rough set-based hybrid fuzzy-neural controller design for industrial wastewater treatment," Water Research, vol. 37, no. 1, pp. 95-107, 2003.

[11] D. Chen, W.X. Zhang, D. Yeung, and E.C.C. Tsang, "Rough approximations on a complete completely distributive lattice with applications to generalized rough sets," Information Sciences, vol. 176, no. 13, pp. 1829-1848, 2006.

[12] S. Chimphlee, N. Salim, M.S.B. Ngadiman, W. Chimphlee, and S. Srinoy, "Independent Component Analysis and Rough Fuzzy based Approach to Web Usage Mining," Proc. Artificial Intelligence and Applications, 2006.

[13] O. Cordon, F. Gomide, F. Herrera, F. Hoffmann, and L. Magdalena, "Ten years of genetic fuzzy systems: current framework and new trends," Fuzzy Sets and Systems, vol. 141, pp. 5-31, 2001.

[14] M. De Cock, C. Cornelis, and E.E. Kerre, "Fuzzy rough sets: beyond the obvious," IEEE International Conference on Fuzzy Systems, vol. 1, pp. 103-108, 2004

[15] M. De Cock and C. Cornelis, "Fuzzy Rough Set Based Web Query Expansion," Proceedings of Rough Sets and Soft Computing in Intelligent Agent and Web Technology, International Workshop at WIIAT2005 (2005 IEEE/WIC/ACM International Joint Conference on Web Intelligence and Intelligent Agent Technology), pp. 9-16, 2005.

[16] D. Dubois and H. Prade, "Rough fuzzy sets and fuzzy rough sets, International Journal of General Systems, vol. 17, pp. 191-209, 1990.

[17] D. Dubois and H. Prade, "Putting Rough Sets and Fuzzy Sets Together," Intelligent Decision Support, pp. 203-232, 1992.

[18] L. Feng, J-J. Qiu, and Y.J. Cao, "Performance of the novel rough fuzzyneural network on short-term load forecasting," Proc. Power Systems Conference and Exposition, pp. 543-547, 2004.

[19] S. Greco, M. Inuiguchi, and R. Slowinski, "Fuzzy rough sets and multiple-premise gradual decision rules," International Journal of Approximate Reasoning, vol. 41, pp. 179-211, 2005.

[20] S. Greco, M. Inuiguchi, and R. Slowinski, "A new proposal for fuzzy rough approximations and gradual decision rule representation," Transactions on Rough Sets II, Lecture Notes in Computer Science, vol. 3135, pp. 319-342, 2004.

[21] L. Han, J. F. Peters, S. Ramanna, and R. Zhai, "Classifying Faults in High Voltage Power Systems: A Rough-Fuzzy Neural Computational Approach," Proc. 7th International Workshop on Rough Sets, Data Mining, and Granular-Soft Computing, pp. 47-54, 1999.

[22] S. Hirano and S. Tsumoto, "Rough Clustering and Its Application to Medicine," Journal of Information Science, vol. 124, pp. 125-137, 2000.

[23] T.P. Hong, Y.L. Liou, and S.L. Wang, "Learning with Hierarchical Quantitative Attributes by Fuzzy Rough Sets," Proc. Joint Conference on Information Sciences, Advances in Intelligent Systems Research, 2006.

[24] F. Hoppner, R. Kruse, F. Klawonn, and T. Runkler, Fuzzy Cluster Analysis: Methods for Classification, Data Analysis and Image Recognition, John Wiley and Sons, 2000.

[25] Q. Hu, D. Yu, and Z. Xie, "Information-preserving hybrid data reduction based on fuzzy-rough techniques," Pattern Recognition Letters, vol. 27, no. 5, pp. 414-423, 2006.

[26] Q. Hu, D. Yu, Z. Xie, and J. Liu, "Fuzzy probabilistic approximation spaces and their information measures," IEEE Transactions on Fuzzy Systems vol. 14, no.2, pp. 191-201, 2006.

[27] Y-X. Huang, C-G. Zhou, S-X. Zou, Y. Wang, and Y-C. Liang, "A Rough-Set-Based Fuzzy-Neural-Network System for Taste Signal Identification," Proc. International Symposium on Neural Networks, pp. 337-343, 2004.

[28] H.H. Huang, Y.H. Kuo, and H.C. Yang, "Fuzzy-Rough Set Aided Sentence Extraction Summarization," Proc. First International Conference on Innovative Computing, Information and Control (ICICIC'06), vol. 1, pp. 450-453, 2006.

[29] R. Jensen and Q. Shen, "Semantics-Preserving Dimensionality Reduction: Rough and Fuzzy-Rough Based Approaches," IEEE Trans. Knowl. Data Eng., vol. 16, no. 12, pp. 1457-1471, 2004. 
[30] R. Jensen and Q. Shen, "Fuzzy-Rough Data Reduction with Ant Colony Optimization," Fuzzy Sets and Systems, vol. 149, no. 1, pp. 5-20, 2005.

[31] R. Jensen and Q. Shen, "Fuzzy-Rough Feature Significance for Fuzzy Decision Trees," Proc. 2005 UK Workshop on Computational Intelligence, pp. 89-96, 2005.

[32] R. Jensen and Q. Shen, "Fuzzy-Rough Sets Assisted Attribute Selection," IEEE Transactions on Fuzzy Systems, vol. 15, no. 1, pp. 73-89, 2007.

[33] L.I. Kuncheva, "Fuzzy rough sets: application to feature selection," Fuzzy Sets and Systems, vol. 51, no. 2, pp. 147-153, 1992.

[34] P. Lingras, "Rough Neural Networks," Proc. International Conference on Information Processing and Management of Uncertainty in Knowledge-Based Systems (IPMU'96), pp. 1445-1450.

[35] P. Lingras, "Unsupervised Learning Using Rough Kohonen Neural Network Classifiers," Proc. Symposium on Modelling, Analysis and Simulation, CESA'96 IMACS Multiconference, pp. 753-757, 1996.

[36] P. Lingras, "Fuzzy-Rough and Rough-Fuzzy Serial Combinations in Neurocomputing," Neurocomputing Journal, vol. 36, pp. 29-44, 2001.

[37] P. Lingras and C. Davies, "Applications of Rough Genetic Algorithms," Computational Intelligence, vol. 17, no. 3, pp. 435-445, 2001.

[38] P. Lingras, M. Hogo, and M. Snorek, "Interval Set Clustering of Web Users using Modified Kohonen Self-Organizing Maps based on the Properties of Rough Sets,"Web Intelligence and Agent Systems, vol. 2, no. 3, pp. 217-230, 2004.

[39] P. Lingras and C. West, "Interval Set Clustering of Web Users with Rough K-means," Journal of Intelligent Information System, vol. 23, no. 1 , pp. 5-16, 2004

[40] P. Lingras, R. Yan, and A. Jain, "Clustering of Web Users: K-Means vs. Fuzzy C-Means," Proc. First Indian International Conference on Artificial Intelligence, pp. 1062-1073, 2003.

[41] N. Mac Parthalain, R. Jensen, and Q. Shen, "Fuzzy Entropy-assisted Fuzzy-Rough Feature Selection," Proc. 2006 IEEE International Conference on Fuzzy Systems, pp. 423-430, 2006.

[42] C-W. Mao, S-H. Liu, and J-S. Lin, "Classification of multispectral images through a rough-fuzzy neural network," Optical engineering, vol. 43, pp. 103-112, 2004.

[43] J.S. Mi and W.X. Zhang, "An axiomatic characterization of a fuzzy generalization of rough sets," Information Sciences, vol. 160, no. 1-4, pp. 235-249, 2004.

[44] P. Mitra and S. Mitra, "Staging of Cervical Cancer with Soft Computing," IEEE Transactions on Biomedical Engineering, vol. 47, no. 7, pp. 934-940, 2000.

[45] N.N. Morsi and M. M. Yakout, "Axiomatics for fuzzy rough sets," Fuzzy Sets and Systems, vol. 100, no. 1-3, pp. 327 - 342, 1998.

[46] S. Nanda and S. Majumdar, "Fuzzy rough sets," Fuzzy Sets and Systems, vol. 45, pp. 157-160, 1992.

[47] S.K. Pal, Pattern Recognition Algorithms for Data Mining, Chapman and Hall, 2004.

[48] S.K. Pal and S. Mitra, Neuro-Fuzzy Pattern Recognition : Methods in Soft Computing, New York: John Wiley, 1999.

[49] S.K. Pal, S. Mitra, and P. Mitra, "Rough-Fuzzy MLP: Modular Evolution, Rule Generation, and Evaluation," IEEE Transactions on Knowledge and Data Engineering, vol. 15, no. 1, pp. 14-25, 2003.

[50] S.K. Pal and A. Skowron (Eds.), Rough-Fuzzy Hybridization: A New Trend in Decision Making, Springer Verlag, Singapore, 1999.

[51] Z. Pawlak, "Rough Sets," International Journal of Information and Computer Sciences vol. 11, pp. 145-172, 1982.

[52] Z. Pawlak, Rough Sets: Theoretical Aspects of Reasoning About Data. Kluwer Academic Publishing, 1991.

[53] W. Pedrycz, "Shadowed sets: bridging fuzzy and rough sets," in [50], pp. 179-199, 1999.

[54] J.F. Peters, A. Skowron, Z. Suraj, W. Rzasa, and M. Borkowski, "Clustering: A rough set approach to constructing information granules," Proc. 6th International Conference on Soft Computing and Distributed Processing, pp. 57-61, 2002.

[55] A. Petrosino and M. Ceccarelli, "Unsupervised Texture Discrimination Based on Rough Fuzzy Sets and Parallel Hierarchical Clustering," Proc IEEE International Conference on Pattern Recognition, pp. 1100-1103, 2000

[56] K. Qina and Z. Pei, "On the topological properties of fuzzy rough sets," Fuzzy Sets and Systems, vol. 151, no. 3, pp. 601-613, 2005.

[57] A.M. Radzikowska and E.E. Kerre, "A comparative study of fuzzy rough sets," Fuzzy Sets and Systems, vol. 126, no. 2, pp. 137-155, 2002 .
[58] A.M. Radzikowska and E.E. Kerre, "Fuzzy Rough Sets Based on Residuated Lattices," Transactions on Rough Sets II, Lecture Notes in Computer Science, vol. 3135/2004, pp. 278-296, 2004.

[59] M. Sarkar, "Fuzzy-Rough nearest neighbors algorithm," Proc. IEEE International Conference on Systems, Man and Cybernetics, pp. 35563561, 2000.

[60] M. Sarkar, "Ruggedness measures of medical time series using fuzzyrough sets and fractals," Pattern Recognition Letters, vol. 27, no. 5, pp. 447-454, 2006

[61] Q. Shen and R. Jensen, "Selecting Informative Features with FuzzyRough Sets and Its Application for Complex Systems Monitoring," Pattern Recognition, vol. 37, no. 7, pp. 1351-1363, 2004.

[62] D. Shan, N. Ishii, Y. Hujun, N. Allinson, R. Freeman, J. Keane, and S. Hubbard, "Feature weights determining of pattern classification by using a rough genetic algorithm with fuzzy similarity measure," Proc. Intelligent data engineering and automated learning, pp. 544550, 2002.

[63] P. Srinivasan, M.E. Ruiz, D.H. Kraft, and J. Chen. "Vocabulary mining for information retrieval: rough sets and fuzzy sets," Information Processing \& Management, vol. 37, no. 1, pp. 15-38. 1998.

[64] H. Thiele, "Fuzzy rough sets versus rough fuzzy sets - an interpretation and a comparative study using concepts of modal logics," Technical report no. CI-30/98, University of Dortmund, 1998.

[65] G.C.Y. Tsang, D. Chen, E.C.C. Tsang, J.W.T. Lee, and D.S. Yeung, "On attributes reduction with fuzzy rough sets," Proc. 2005 IEEE International Conference on Systems, Man and Cybernetics, vol. 3, pp. 2775-2780, 2005

[66] K.E. Voges, N.K.L. Pope, and M.R. Brown, "Cluster Analysis of Marketing Data: A Comparison of K-Means, Rough Set, and Rough Genetic Approaches," in Heuristics and Optimization for Knowledge Discovery, Edited by H.A. Abbas, R.A. Sarker, C.S. Newton, Idea Group Publishing, pp. 208-216, 2002.

[67] X. Wang, J. Yang, X. Teng and N. Peng, "Fuzzy-Rough Set Based Nearest Neighbor Clustering Classification Algorithm," Lecture Notes in Computer Science, vol. 3613/2005, pp. 370-373, 2005.

[68] X.Z. Wang, Y. Ha, and D. Chen, "On the reduction of fuzzy rough sets," Proc. 2005 International Conference on Machine Learning and Cybernetics, vol. 5, pp. 3174-3178, 2005.

[69] Z. Wang, X. Shao, G. Zhang, and H. Zhu, "Integration of Variable Precision Rough Set and Fuzzy Clustering: An Application to Knowledge Acquisition for Manufacturing Process Planning," Proc. 10th International Conference, RSFDGrC 2005, pp. 585-593, 2005.

[70] A. Wojna, "Analogy-Based Reasoning in Classifier Construction," Transactions of Rough Sets, vol. 4, pp. 277-374, 2005.

[71] W.Z. Wu, J.S. Mi, and W.X. Zhang, "Generalized fuzzy rough sets," Information Sciences, vol. 151, pp. 263-282, 2003.

[72] W.Z. Wu and W.X. Zhang, "Constructive and axiomatic approaches of fuzzy approximation operators," Information Sciences, vol. 159, no.3-4, pp. 233-254, 2004

[73] W.Z. Wu, "A Study on Relationship Between Fuzzy Rough Approximation Operators and Fuzzy Topological Spaces," L. Wang and Y. Jin (Eds.): FSKD 2005, LNAI 3613, pp. 167-174, 2005.

[74] W.Z. Wu, Y. Leung, and J.S. Mi, "On characterizations of ( $I, T)$-fuzzy rough approximation operators," Fuzzy Sets and Systems, vol. 154, no. 1, pp. 76-102, 2005

[75] M. Wygralak, "Rough sets and fuzzy sets - some remarks on interrelations," Fuzzy Sets and Systems, vol. 29, no. 2, pp. 241-243, 1989.

[76] Y.Y. Yao, "Combination of rough and fuzzy sets based on $\alpha$-level sets," in: T.Y. Lin, N. Cereone (Eds.), Rough Sets and Data Mining: Analysis of Imprecise Data, Kluwer Academic Publishers, pp. 301-321, 1997.

[77] D.S. Yeung, D. Chen, E.C.C. Tsang, J.W.T. Lee, and W. Xizhao, "On the Generalization of Fuzzy Rough Sets," IEEE Transactions on Fuzzy Systems, vol. 13, no. 3, pp. 343-361, 2005

[78] L. Zadeh, "Fuzzy sets," Information and Control, vol. 8, no. 3, pp. 338-353, 1965.

[79] J. Zhang, "Fuzzy neural networks based on rough sets for process modeling," Proc. Fifth International Symposium on Instrumentation and Control Technology, pp. 844-847, 2003.

[80] Y. Zhao, X. Zhou, and G. Tang, "A rough set-based fuzzy clustering," Proc. Second Asia information retrieval symposium, pp. 401-409, 2005.

[81] L. Zhou, W. Li and Y. Wu, "Face Recognition Based on Fuzzy Rough Set Reduction," Proc. 2006 International Conference on Hybrid Information Technology (ICHIT'06), vol. 1, pp. 642-646, 2006. 